\title{
Pathogenic characteristics of sputum and bronchoalveolar lavage fluid samples from patients with lower respiratory tract infection
}

\section{Zheng Peng}

Tongji Hospital of Tongji Medical College of Huazhong University of Science and Technology Jin'an Zhou

Tongji Hospital of Tongji Medical College of Huazhong University of Science and Technology lei tian ( $\square$ iso15189@126.com )

Tongji Hospital of Tongji Medical College of Huazhong University of Science and Technology

\section{Research article}

Keywords: sputum, respiratory tract infection, bronchoscopic lavage fluid, MRSA, CR-KPN

Posted Date: June 7th, 2020

DOI: https://doi.org/10.21203/rs.3.rs-32101/v1

License: (c) (i) This work is licensed under a Creative Commons Attribution 4.0 International License.

Read Full License 


\section{Abstract}

Background: Lower respiratory tract infections $\llbracket$ LRIs $₫$ were a very common disease, no matter in community acquired infection or hospital acquired infection. Sputum and bronchoalveolar lavage fluid (BALF) were the most important specimens of LRIs. The choice of antibiotics for the treatment of LRIs usually depended on the results of antimicrobial sensitivity of bacteria isolated from sputum and BALF. However, it was rarely reported to compare the pathogens isolated from sputum and BALF and the difference of antimicrobial sensitivity.

Methods: A retrospective study was conducted to analyze the differences between sputum and BALF samples in pathogen isolation and antimicrobial sensitivity in our hospital.

Results: In our hospital during 2013-2015 year, the quality evaluation of sputum samples was not conducted before sputum culture, but in 2016-2018 year, the quality evaluation of sputum samples was conducted firstly and only qualified sputum samples were cultured. The results of pathogen culture showed that Pseudomonas aeruginosa, Acinetobacter baumannii, Klebsiella pneumoniae, Staphylococcus aureus and Haemophilus influenzae were the top five pathogens isolated from sputum and BALF. Antimicrobial susceptibility test showed for these five pathogens the susceptibility rates of BALF isolates to most antibiotics were higher than those isolated from sputum. The sensitivity of $A$. baumannii to common antibiotics was less than $50 \%$. In particular, the detection rate of carbapenem resistant $A$. baumannii (CR-ABA) in sputum and BALF was higher than $80 \%$. The sensitivity of $P$. aeruginosa to antibiotics other than ticarcillin/clavulanic acid and minocycline were higher than $50 \%$. The detection rate of carbapenem resistant Klebsiella pneumoniae (CR-KPN) was 10\% - 20\% in 2013-2015 and $30 \%-50 \%$ in $2016-2018$. The detection rate of MRSA in sputum was higher than $80 \%$, while that in BALF was $65 \%-70 \%$. The separation rate of beta-lactamase-negative ampicillin-resistant $H$. influenzae (BLNAR) was between 0-6\%.

Conclusions: The sensitivity of strains isolated from BALF to commonly used antibiotics was generally higher than that from sputum. MRSA, CR-KPN and A. baumannii were the focus of infection control for LRIs.

\section{Background}

Lower respiratory tract infections (LRIs) were the most common infectious disease of respiratory tract [12]. Irrational use of antibiotics would delay the patient's condition and cause serious bacterial resistance [1-2]. According to the data from China Antimicrobial Resistance Surveillance System (CARSS) in 2015, the major specimen type from inpatients in respiratory departments in China were sputum $(81.6 \%$, $41,131 / 50,417)[1-2]$. Due to the convenience of specimen collection, sputum had always been the most common type of specimens in clinical microbiology laboratories in China. But easily confused by oral colonization flora, it was difficult to judge the results of sputum culture as infectious, colonized or contaminated bacteria. For the results of sputum culture, it was difficult for clinicians to evaluate the 
significance, and even more difficult for laboratory physicians. BALF cultures have been shown to be a reliable method for determining the bacterial etiology of LRIs [3]. As the largest number of sputum samples, whether there is difference between the culture results of sputum samples and BALF culture results and how big the difference was, the research on this question was rarely reported.

In our hospital, there was no quality evaluation of sputum samples before 2015 . However, since 2016, we have carried out the quality evaluation of sputum samples. Only qualified sputum samples can be used for sputum culture. Now it was well known that unqualified sputum specimens, such as saliva, have no significance in bacterial culture. And the industry had reached a consensus that sputum culture without microscopic examination was of no value [4]. Here, we compare the bacterial culture results of sputum and BALF in 2013-2015 and 2016-2018 in our hospital to explore the differences between sputum and BALF in isolation of pathogenic bacteria, so as to provide a better reference for clinical treatment.

\section{Materials And Methods}

\section{Study design and procedures}

Sputum culture and BALF culture results from 2013-2015 and 2016-2018 in our hospital were analyzed retrospectively. The most common pathogens isolated from sputum and BALF in 2013-2015 and 20162018 were compared. The sensitivity of the same pathogens from sputum and BALF to commonly used antibiotics was compared. All specimens (Including sputum and BALF) were taken from the clinical departments of Tongji Hospital and sent to department of laboratory medicine. Isolation and antimicrobial sensitivity test of pathogenic bacteria were carried out in clinical microbiology laboratory according to standardized operation.

\section{Interpretation criteria for qualified sputum specimens}

The qualified interpretation of sputum specimens in this study is based on the Chinese standard [5]. Since 2016, smear microscopy has been required for each specimen requiring sputum culture. Sputum specimens satisfying the following three conditions will be treated as qualified specimens. First, a specimen with $\geq 25$ white blood cells (WBC) per average low-power field (LPF) and squamous epithelial cells $(E P I)<25$ per LPF. Second, the ratio of WBC to EPI was more than 10:1, and the single-form bacteria were predominant. Third, EPI $<10$ per LPF, and alveolar macrophages and columnar epithelial cells existed. In addition to the above three cases, when EPI> 10 per LPF, they were considered to be unqualified sputum specimens.

\section{Identification of strains and antimicrobial susceptibility test}

For isolates from the same position of the same patient, only the first isolate was included in the analysis according to CLSI M39 [6]. The identification of strains was carried out by biochemical experiments, automatic identification system (Vitek-2-compact, BioMerier Products) and/or IVD-MALDI Biotyper (Bruker, Germany). Antimicrobial susceptibility test was carried out and explained according to CLSI 2018 
by disk diffusion method and E test method. [7]. ATCC 25922, 25923, 27853, 49247,49619, 90028, 35218, 700603,29213 were used for quality control of indoor antimicrobial sensitivity tests, which were performed weekly. According to CLSI M39, the drug sensitivity results of different antimicrobial agents were expressed by sensitivity rate [6].

\section{Statistical analysis}

All patient and strain information were stored in the WHONET software. WHONET 5.6 software was used to analyze antimicrobial susceptibility data.

\section{Results}

\section{Pathogenic spectrum of LRIs}

The number of bacteria isolated from sputum and BALF in 2013-2015 was 12957 and 848, respectively, compared with 6740 and 2239 in 2016-2018. In 2013-2015 and 2016-2018, the top five pathogens isolated from sputum and BALF were $S$. aureus, P. aeruginosa, A. baumannii, K. pneumoniae and $H$. influenzae. $H$. influenzae was always the fifth, the other four were in different order of sputum and BALF (Figure 1).

\section{Antimicrobial susceptibility}

The susceptibility rates of $P$. aeruginosa in sputum and BALF to commonly used antibiotics were compared in (2013-2015) and (2016-2018). The sensitivity of $P$. aeruginosa in BALF to commonly used antibiotics was higher than that of sputum isolates, whether in 2013-2015 or 2016-2018. Except for ticarcillin/clavulanic acid and minocycline, the sensitivity of $P$. aeruginosa to commonly used antibiotics was more than $50 \%$. The sensitivity of sputum isolates to ticarcillin/clavulanic acid and minocycline was $10 \%-20 \%$, while that of BALF isolates was $30 \%-40 \%$ (Figure 2 ).

The susceptibility rate of $A$. baumannii to commonly used antibiotics showed that the susceptibility rate of isolates from BALF in 2016-2018 was higher than that of sputum isolates, except minocycline, tegacycline and piperacillin. However, data from 2013 to 2015 showed that the susceptibility rates of sputum isolates to cefoperazone sulbactam, ampicillin sulbactam, imipenem, meropenem, amikacin, gentamicin, tobramycin, ciprofloxacin and tigacycline were higher than those of BALF isolates. The sensitivity of $A$. baumannii to commonly used antibiotics was low. The sensitivity of $A$. baumannii to minocycline and tegacyclin was only $30 \%-50 \%$, the others are almost lower than $30 \%$ (Figure 3 ).

The sensitivity analysis of $K$. pneumoniae to commonly used antibiotics showed that the sensitivity of BALF isolates was higher than that of sputum isolates, whether in 2013-2015 or 2016-2018. From 20132015 to 2016-2018, the sensitivity of $K$. pneumoniae to commonly used antimicrobial agents was decreasing, whether it was isolated from sputum or BALF. Tegacyclin, amikacin, imipenem and meropenem were the most sensitive drugs (Figure 4). 
The susceptibility rate of $S$. aureus to commonly used antibiotics showed that the susceptibility rate of BALF isolates was higher than that of sputum isolates in 2016-2018. However, in 2013-2015, except erythromycin, clindamycin and trimethoprim/sulfamethoxazole, the sensitivity of BALF isolates was higher than that of sputum isolates. Linezolid, vancomycin, teicoplanin, tegacyclin and Trimethoprim sulfamethoxazole were the most sensitive drugs to $S$. aureus (Figure 5).

The sensitivity rate of $H$. influenzae to antibiotics other than Trimethoprim sulfamethoxazole was almost more than $60 \%$. The sensitivity rates of $H$. influenzae isolated from sputum and BALF to Trimethoprim sulfamethoxazole were $30 \%$ - $50 \%$ (Figure 6).

The analysis of specific resistant phenotypes showed that the ESBLs producing $K$. pneumoniae was decreasing, while the $C R-K P N$ was increasing. The detection rate of $C R-A B A$ remained at a high level, all of which were more than $60 \%$. Different from $C R-A B A$, the detection rates of $C R-P A E$ were lower than $40 \%$. The detection rate of MRSA remained at a high level, all of which were higher than $65 \%$. For $H$. influenzae, the detection rates of BLNAR were lower than $6 \%$ (Table 1).

\section{Discussion}

This study found that the main pathogens isolated from sputum and BALF were $P$. aeruginosa, $A$. baumannii, K. pneumoniae, S. aureus and $H$. influenzae. However, the drug resistance rate of sputum and BALF strains to commonly used antibiotics was different. The sensitivity of BALF isolates to common antibiotics was higher than that of sputum isolates almost.

The clinical significance of sputum culture had always been controversial. Due to the influence of oral colonization bacteria, it was difficult to judge the pathogenic bacteria of sputum culture as infection bacteria or colonization bacteria. How to evaluate the significance of sputum culture had always been a puzzling problem. In this study, we evaluated the significance of sputum culture from a new perspective. This study compared the difference of pathogen spectrum and antimicrobial sensitivity between sputum samples and BALF, and compared the difference before and after quality control of sputum samples. Studies had shown that, even for qualified sputum specimens, the results of culture and antimicrobial sensitivity were still quite different from those of BALF. BALF was obtained through fiberoptic bronchoscope and could represent the status of lower respiratory tract infection. However, sputum specimens were easily contaminated by colonies in the upper respiratory tract. Therefore, clinicians needed to be very careful in diagnosing and treating lower respiratory tract infections based on the results of sputum culture.

Sputum specimens were not a good type of specimens, from the point of view of diagnosis of LRIs. Doctors obtained biopsy specimens through fiberoptic bronchoscopy, which could represent LRIs. But after all, it belonged to the invasive operation and was not suitable for every patient. At present, qualified sputum specimens, together with some invasive surgical specimens (transtracheal aspiration, bronchoalveolar lavage, protected brush samples, etc) were acceptable in the global LRIs surveillance project [8]. In the face of LRIs, what kind of specimen to send was another difficult problem. The 
American Association of Pediatric Infectious Diseases told us that blood cultures should be sent for moderate to severe community-acquired pneumonia in children, especially complex pneumonia [9]. But at present, the rate of blood culture in Chinese patients with LRIs was not high. A multicenter study from China showed that blood culture isolates accounted for only $5.3 \%$ of all specimen types [1-2]. For LRIs, should we do blood culture or sputum culture? The Lancet journal gave us the answer. Different strategies should be adopted for different types of patients. For outpatient blood culture and sputum culture was not needed routinely. For inpatients with low severity, only sputum culture was needed. For inpatient with moderate severity and no ICU sputum culture, blood culture, legionella urinary antigen and pneumococcal urinary antigen should be adopted routinely. For inpatient in ICU with high severity, invasive sampling should also be performed in addition to all the above tests [10].

The results of this study showed that $A$. baumannii was less sensitive to common antibiotics, except for tegacyclin and minocycline, which were more than $30 \%$. Our results were consistent with those of China antimicrobial surveillance network (CHINET) in 2018, which indicated that 16566 strains of $A$. baumannii from the respiratory tract were more than $50 \%$ resistant to antibiotics other than tegacyclin, minocycline and polymyxin [11]. Different from A. baumannii, P. aeruginosa was more sensitive to common antimicrobial agents, except ticarcillin clavulanic acid and minocycline, which were more than $50 \%$. This is consistent with previously reported data from China [1-2,11]. For K. pneumoniae, although ESBLs producing strain was decreasing, CR-KPN was increasing significantly. In addition, some studies have shown that CR-KPN with high virulence may be spread in hospital [12]. The increase of CR-KPN in the world has brought difficulties to infection control. The treatment of MRSA still depends on glycopeptide drugs, tegacyclin and Trimethoprim sulfamethoxazole. The sensitivity of $H$. influenzae to common antibiotics was high, and the detection rate of BLNAR in this area was lower than that reported in Japan [13].

There were several limitations in the studies. First, in this study, no distinction was made between natural expectoration, induced sputum and sputum aspiration. Second, whether BALF was a qualified sample had not been judged in this study. We hoped that in future studies, BALF would be interpreted as qualified as sputum specimens and evaluation of clinical value of various respiratory tract specimens would be carried out.

\section{Conclusions}

Although the main pathogens isolated from sputum and BALF were the same, the drug sensitivity results were different. The sensitivity of BALF isolate was higher than that of sputum. The control of MRSA, CR$\mathrm{KPN}$ and $A$. baumannii was still the focus of lower respiratory tract infection control.

\section{Abbreviations}


LRIs: lower respiratory tract infections, CARSS, China Antimicrobial Resistance Surveillance System; WBC, white blood cells; LPF, low-power field; EPI, squamous epithelial cells; BALF, bronchoscopic lavage fluid; CR-ABA: Acinetobacter baumannii resistant to carbapenems; CR-KPN: Klebsiella pneumoniae resistant to carbapenems; BLNAR: beta-lactamase-negative ampicillin-resistant $H$. influenzae; CHINET: China antimicrobial surveillance network

\section{Declarations}

\section{Ethics approval and consent to participate}

The study protocol was approved by the Tongji Hospital ethics committee for research in health. The Tongji Hospital ethics committee also approved the waiver of informed consent to participate in this study due to its retrospective design. All patient data were anonymous prior to the analysis.

\section{Consent to publish}

Not applicable.

\section{Availability of data and materials}

The datasets used and/or analyzed during the current study are available from the corresponding author on reasonable request.

\section{Competing interests}

The authors declare that they have no competing interest.

\section{Author Contributions}

LT designed the study. LT analyzed the data and drafted the manuscript. ZP and JZ revised the manuscript. All authors reviewed the manuscript prior to submission.

\section{Acknowledgments}

We thank all members of laboratory medicine department of TongJi hospital for their participation in these studies.

\section{Authors' information}

Lei Tian and Zheng Peng were from Department of Clinical Laboratory, Tongji Hospital, Tongji Medical College, Huazhong University of Science and Technology, Wuhan, Hubei Province, China. Jin'an Zhou was from Department of Blood Transfusion, Tongji Hospital, Tongji Medical College, Huazhong University of Science and Technology, Wuhan, Hubei Province, China.

\section{References}


1. Tang $X$, Xiao $M$, Zhuo $C, X u Y, Z$ hong N: Multi-level analysis of bacteria isolated from inpatients in respiratory departments in China. J Thorac Dis 2018, 10(5):2666-2675.

2. Tang $X$, Zhuo $C$, Xu YC, Zhong NS: [The composition and antimicrobial resistance of isolates from lower respiratory tract and blood in hospitalized patients in respiratory ward: a multicenter national study in China]. Zhonghua Jie He He Hu Xi Za Zhi 2018, 41(4):281-287.

3. Escribano Montaner A, Garcia de Lomas J, Villa Asensi JR, Asensio de la Cruz O, de la Serna Blazquez O, Santiago Burruchaga M, Mondejar Lopez P, Torrent Vernetta A, Feng Y, Van Dyke MK et al: Bacteria from bronchoalveolar lavage fluid from children with suspected chronic lower respiratory tract infection: results from a multi-center, cross-sectional study in Spain. Eur J Pediatr 2018, 177(2):181-192.

4. Heineman HS, Chawla JK, Lopton WM: Misinformation from sputum cultures without microscopic examination. J Clin Microbio/ 1977, 6(5):518-527.

5. Health and Family Planning Commission. Performance guidelines for bacterials culture of lower respiratory tract infections. WS/T 499, GB,GBT,GB/T Chinese Standard; 2017.

6. Clinical and Laboratory Standards Institute. Analysis and presentation of cumulative antimicrobial susceptibility test data, M39-A4. Wayne, PA: Clin Lab Stand Institute; 2014.

7. Clinical and Laboratory Standards Institute. Performance Standards for Antimicrobial Susceptibility Testing, Twenty-eighth Informational Supplement, M100-S28. Wayne, PA: Clin Lab Stand Institute; 2018..

8. Sader HS, Castanheira M, Arends SJR, Goossens H, Flamm RK: Geographical and temporal variation in the frequency and antimicrobial susceptibility of bacteria isolated from patients hospitalized with bacterial pneumonia: results from 20 years of the SENTRY Antimicrobial Surveillance Program (1997-2016). J Antimicrob Chemother 2019, 74(6):1595-1606.

9. Bradley JS, Byington CL, Shah SS, Alverson B, Carter ER, Harrison C, Kaplan SL, Mace SE, McCracken $\mathrm{GH}, \mathrm{Jr}$., Moore MR et al: The management of community-acquired pneumonia in infants and children older than 3 months of age: clinical practice guidelines by the Pediatric Infectious Diseases Society and the Infectious Diseases Society of America. Clin Infect Dis 2011, 53(7):e25-76.

10. Prina E, Ranzani OT, Torres A: Community-acquired pneumonia. Lancet 2015, 386(9998):1097-1108.

11. Hu F, Guo Y, Yang Y, Zheng Y, Wu S, Jiang X, Zhu D, Wang F, China Antimicrobial Surveillance Network Study G: Resistance reported from China antimicrobial surveillance network (CHINET) in 2018. Eur J Clin Microbiol Infect Dis 2019, 38(12):2275-2281.

12. Li J, Huang ZY, Yu T, Tao XY, Hu YM, Wang HC, Zou MX: Isolation and characterization of a sequence type 25 carbapenem-resistant hypervirulent Klebsiella pneumoniae from the mid-south region of China. BMC Microbio/ 2019, 19(1):219.

13. Yanagihara K, Matsumoto T, Aoki N, Sato J, Wakamura T, Kiyota H, Tateda K, Hanaki H, Ohsaki Y, Fujiuchi $S$ et al: Nationwide surveillance of bacterial respiratory pathogens conducted by the surveillance committee of Japanese Society of Chemotherapy, the Japanese Association for 
Infectious Diseases, and the Japanese Society for clinical microbiology in 2014: General view of the pathogens' antibacterial susceptibility. J Infect Chemother 2019, 25(9):657-668.

\section{Table 1}

Table 1. Distribution of special resistant strains

\begin{tabular}{lllll}
\hline & \multicolumn{2}{c}{$\begin{array}{c}2013-2015 \\
\text { bronchoscopic lavage fluid }\end{array}$} & sputum & $\begin{array}{c}2016-2018 \\
\text { bronchoscopic lavage fluid }\end{array}$ \\
\hline iBLs (+) KPN & $50.4 \% \square 785 / 1559 \square$ & $32.8 \% \square 38 / 116 \square$ & $21.5 \% \square 177 / 822 \square$ & $11.4 \% \square 45 / 394 \square$ \\
2-KPN & $19.1 \%(298 / 1559)$ & $10.3 \%(12 / 116)$ & $59.9 \%(492 / 822)$ & $33.0 \%(130 / 394)$ \\
2-ABA & $82.2 \%(2082 / 2534)$ & $65.4 \%(102 / 156)$ & $94.2 \%(1568 / 1664)$ & $89.7 \%(280 / 312)$ \\
2-PAE & $38.2 \%(653 / 1710)$ & $12.2 \%(20 / 164)$ & $37.9 \%(404 / 1066)$ & $23.6 \%(93 / 394)$ \\
RSA & $81.2 \%(2284 / 2813)$ & $69.2 \%(74 / 107)$ & $92.3 \%(1011 / 1195)$ & $67.9 \%(169 / 249)$ \\
.NAR & $5.3 \%(38 / 721)$ & $1.7 \%(1 / 59)$ & $1.2 \%(6 / 491)$ & $0(0 / 170)$ \\
\hline
\end{tabular}

Note: ESBLs (+) KPN: Klebsiella pneumoniae producing extended spectrum $\beta$ lactamase, CR-KPN: Klebsiella pneumoniae resistant to carbapenems, CR-ABA: Acinetobacter baumannii resistant to carbapenems, CR-PAE: Pseudomonas aeruginosa resistant to carbapenems, MRSA: Methicillin resistant Staphylococcus aureus, BLNAR: Haemophilus influenzae with $\beta$ - lactamase negative resistance to ampicillin

\section{Figures}

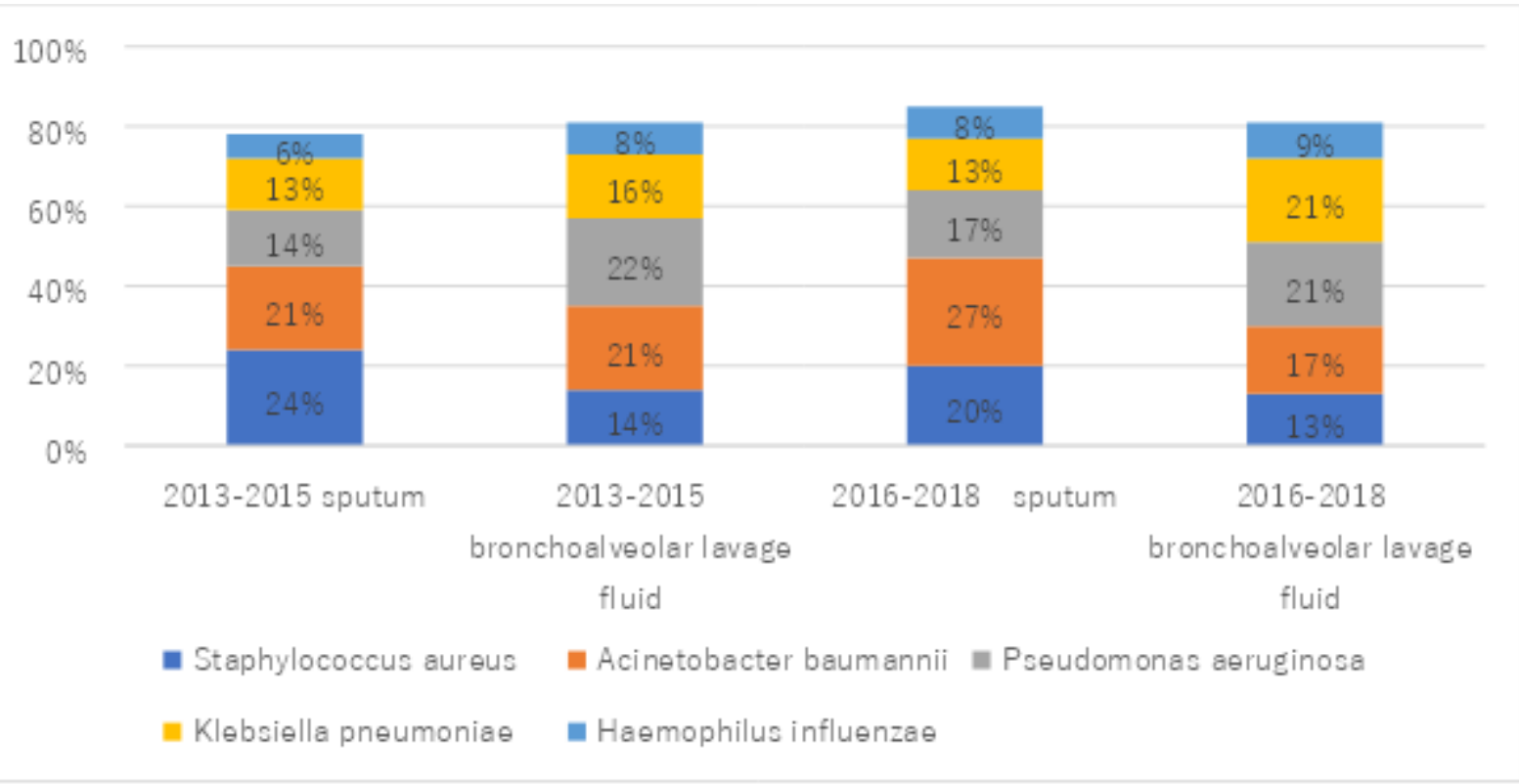

\section{Figure 1}

The main pathogens (top five) isolated from sputum and BALF samples were in 2013-2015 and 20162018. 


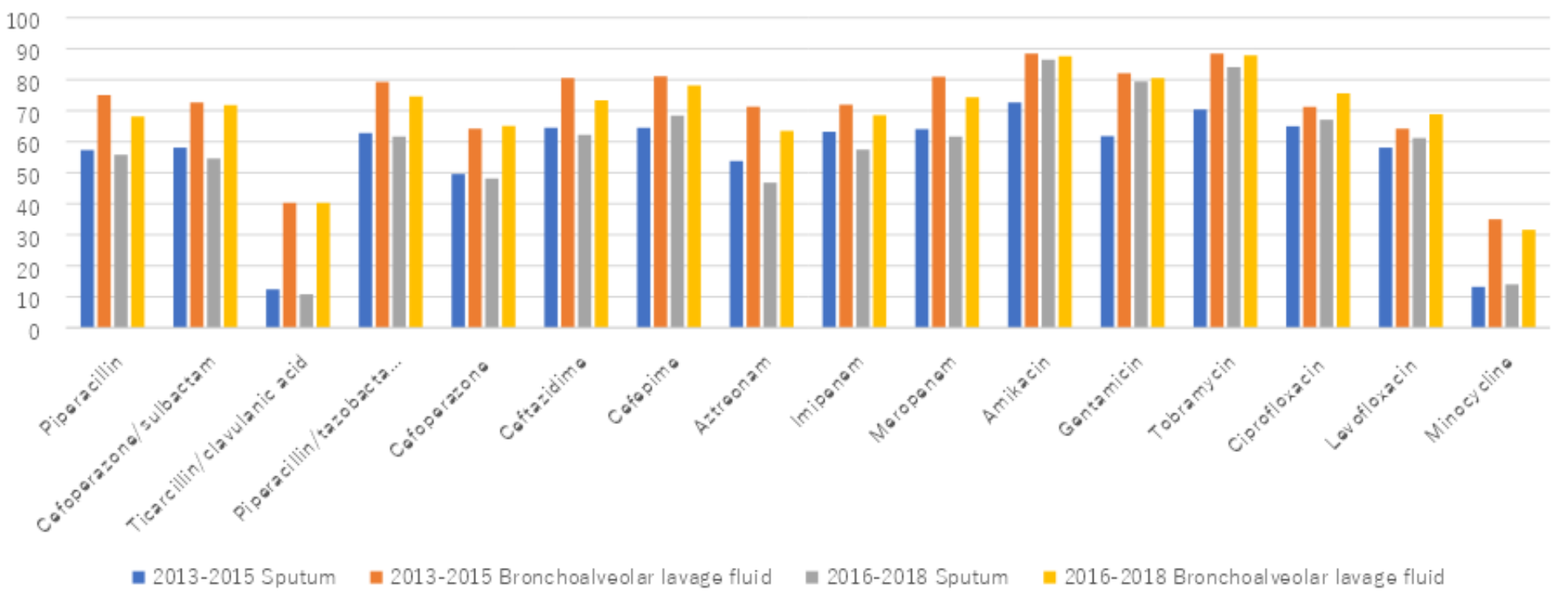

\section{Figure 2}

Sensitivity rate of Pseudomonas aeruginosa isolated from sputum and bronchoalveolar lavage fluid to commonly used antibiotics

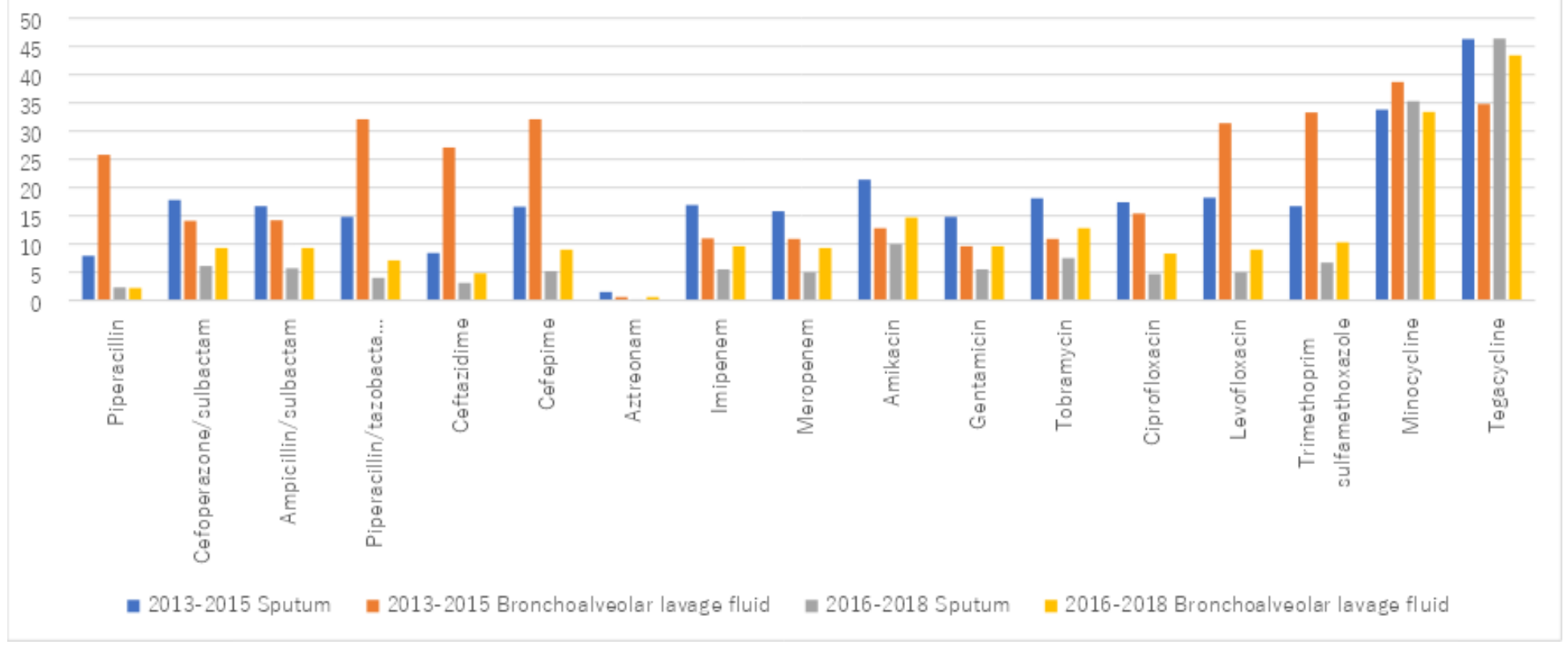

\section{Figure 3}

Sensitivity rate of Acinetobacter baumannii isolated from sputum and bronchoalveolar lavage fluid to commonly used antibiotics 


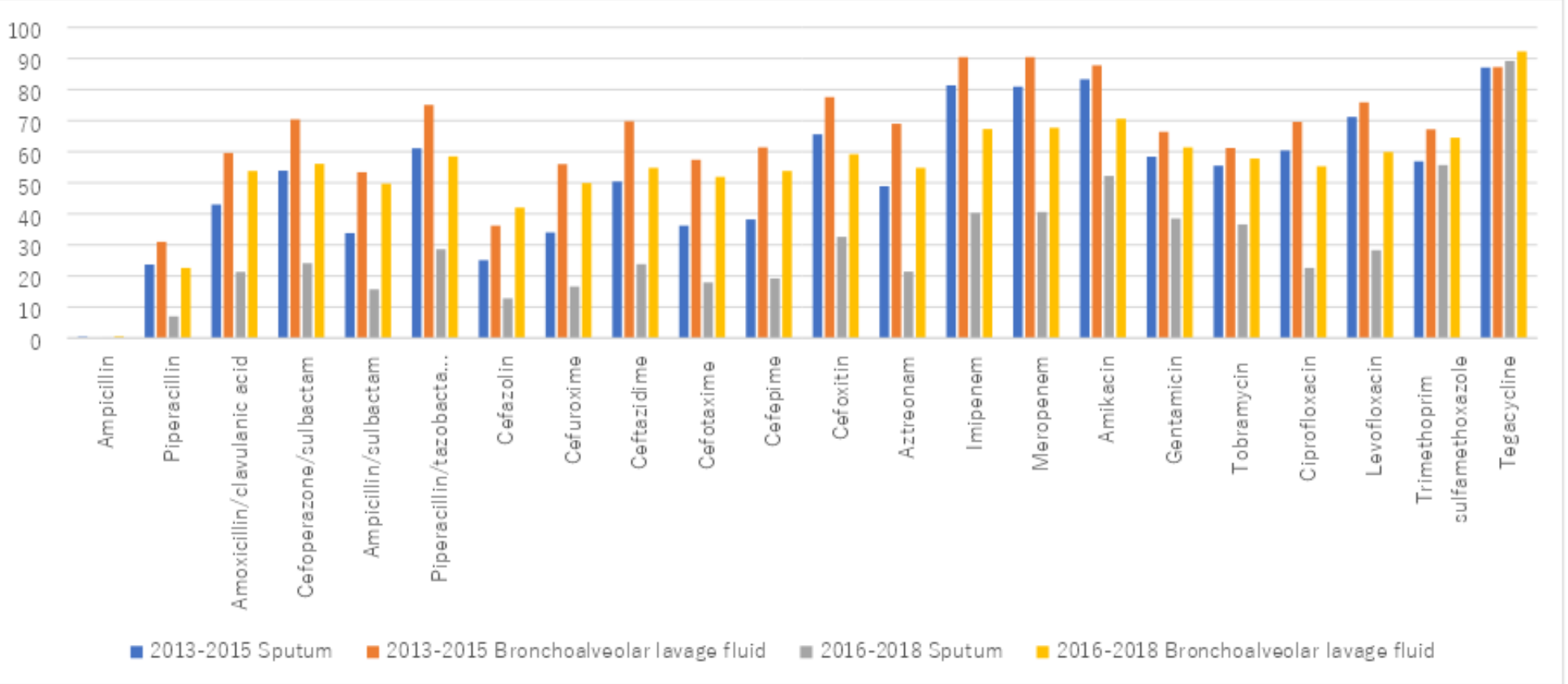

\section{Figure 4}

Sensitivity rate of Klebsiella pneumoniae isolated from sputum and bronchoalveolar lavage fluid to commonly used antibiotics

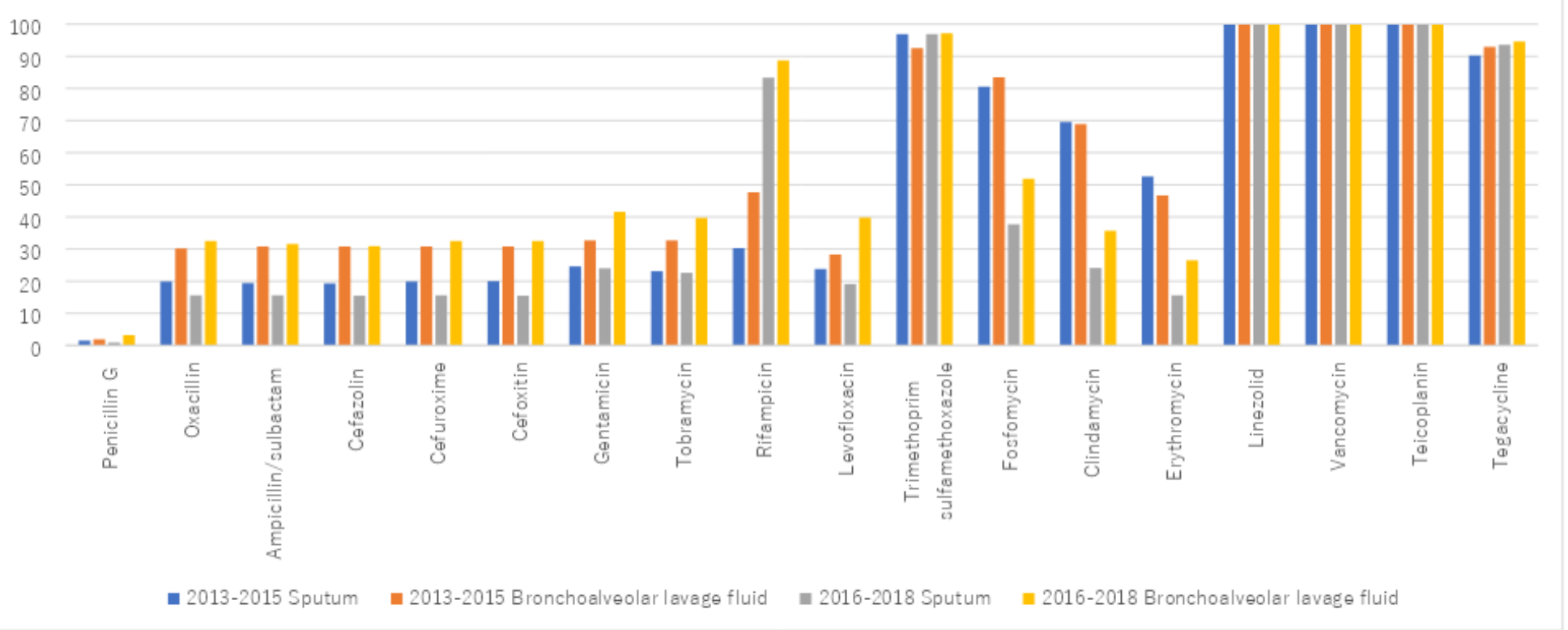

Figure 5

Sensitivity rate of Staphylococcus aureus isolated from sputum and bronchoalveolar lavage fluid to commonly used antibiotics 


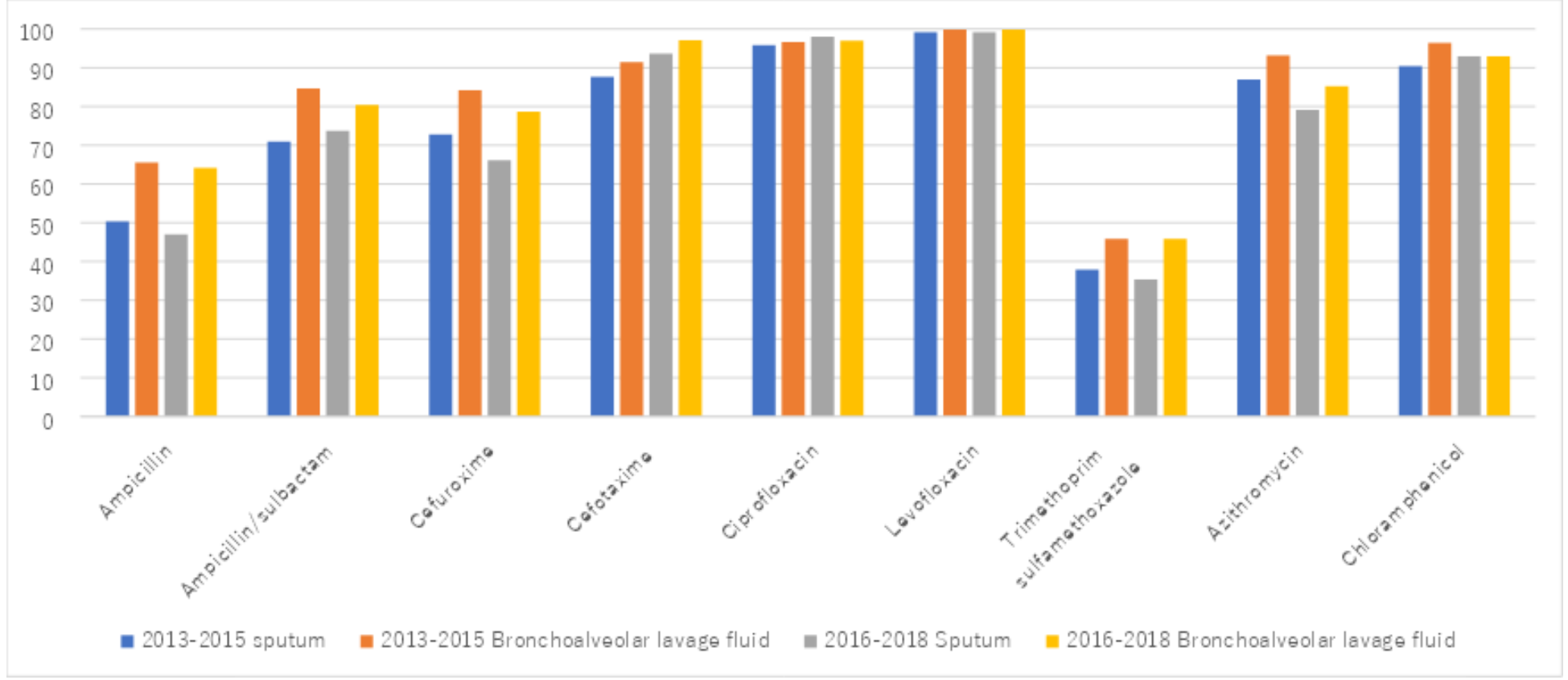

Figure 6

Sensitivity rate of Haemophilus influenzae isolated from sputum and bronchoalveolar lavage fluid to commonly used antibiotics 